
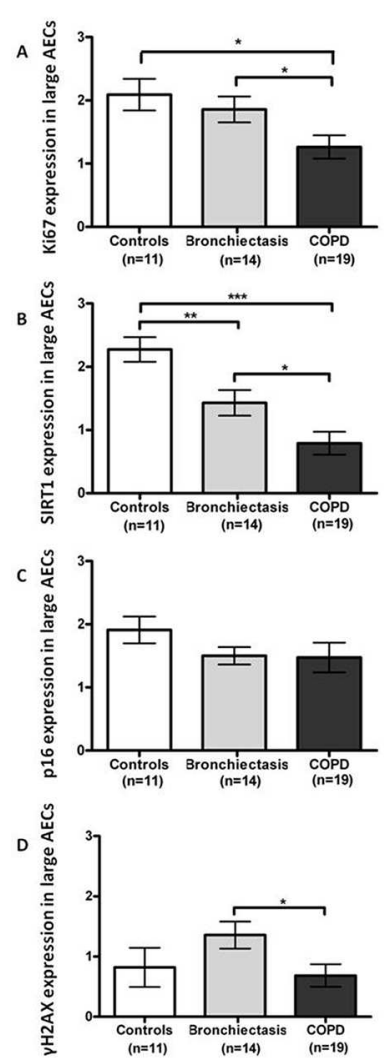

Abstract S65 Figure 1. Expression of Ki67 (A), SIRT1 (B), p16 (C) and $\gamma \mathrm{H} 2 \mathrm{AX}$ (D) in large AECs and small AECs (E, F, G and H, respectively). Results are expressed as mean \pm SEM. Statistics: Kruskal-Wallis and Mann-Whitney $U$ test. ${ }^{*} \mathbf{p}<0.05, * * \mathbf{p}<$ $0.01, * * *$ p $<0.001$.

Results In large AECs, expression of the proliferation marker ki67 and the anti-ageing protein sirtuin 1 (SIRT1) was decreased in COPD as compared to bronchiectasis and controls. There was no difference in expression of the cell-cycle inhibitor p16 and the DNA damage associated foci ?H2AX between the three groups. In small AECs, SIRT1 was decreased in COPD compared to controls and p16 and ?H2AX were increased. Here, ki67 expression did not differ between groups. In bronchiectasis, there was no significant change in senescence marker expression compared to controls, with the exception of decreased SIRT1 in large AECs. Marker expression was not significantly correlated with $\mathrm{FEV}_{1}$ or smoking history. Preliminary work suggests potential co-localisation of ?H2AX at telomeres with ongoing analysis underway.

Conclusions Differential expression of senescence-associated markers between large and small airways in COPD may reflect the distinct patterns of inflammation and functional impairment occurring in the two airway compartments. There is some evidence suggesting a role for senescence in bronchiectasis, though this is less clear than for COPD. Further markers need to be investigated.

\section{S66 TARGETING ANTI-AGEING MOLECULE AMPK RESTORES CORTICOSTEROID SENSITIVITY IN COPD}

AA Azam, PJ Barnes, NM Mercado; Imperial College London, London, UK

10.1136/thoraxjnl-2013-204457.73

Rationale Chronic obstructive pulmonary disease (COPD) is an irreversible inflammatory lung disease and is currently the fourth greatest burden of disease of worldwide. However a key issue is that patients show a lack of response to corticosteroid treatment. Corticosteroid insensitivity is mainly caused by oxidative stress which directly stimulates inflammatory transcription factors and reduces the activity of co-adaptor proteins essential for the inhibitory actions of corticosteroids. AMP-activated kinase (AMPK) is a serine/threonine protein kinase that regulates cellular energy homeostasis and anti-oxidant defences, and has recently been labelled as an anti-ageing molecule. We hypothesised that activation of AMPK using Quercetin reverses corticosteroid resistance caused by cigarette smoke extract (CSE) in a monocytic cell line.

Methods Human monocytic cell line, U937s, were initially incubated with Quercetin $(20 \mu \mathrm{M})$ for 24 hours and then exposed to CSE for 2 hours. Cells were then treated with dexamethasone $\left(1 \times 10^{-11}\right.$ to $\left.1 \times 10^{-6} \mathrm{M}\right)$ for 45 minutes and stimulated with TNF-a $(10 \mathrm{ng} / \mathrm{ml})$ for 24 hours. Supernatants were collected and CXCL8 was measured using ELISA. Corticosteroid resistance was calculated as the ability of dexamethasone to inhibit 25\% of TNFa-induced CXCL-8 $\left(\mathrm{IC}_{25}\right)$. Activation of AMPK by Quercetin was measured using the levels of the phosphorylated AMPK by Western Blot. Nuclear factor erythroid related factor 2 (Nrf-2) levels and glucocorticoid receptor (GR) nuclear translocation were also assessed using Western Blot.

Results CSE induced corticosteroid resistance in U937s $\left(\mathrm{IC}_{25}=30 \mathrm{nM}\right.$ vs $\left.\mathrm{IC}_{25}=5 \mathrm{nM}\right)$. Interestingly Quercetin restored corticosteroid sensitivity by approximately 3 fold $\left(\mathrm{IC}_{25}=\right.$ $11 \mathrm{nM}$ ) compared to CSE. Quercetin increased levels of activated AMPK and also up-regulated the expression of Nrf-2. However, Quercetin was unable to restore GR nuclear translocation.

Conclusions Quercetin was found to be a potential novel therapy for restoration of corticosteroid sensitivity in COPD. Although the mechanism of action remains to be elucidated, Nrf-2 and AMPK activations which increase anti-oxidant levels and prevents oxidative damage could be a key the mechanism of action Activation of AMPK could therefore be a potential novel mechanism for the restoration of corticosteroid sensitivity and Quercetin could be used as an add-on treatment to corticosteroids in COPD.

\section{Delivering better, safer care}

\section{S67 COPD-IN THE NEWS!}

LE Hodgson, S Bax, M Montefort, JP Zahra, R Venn, H Ranu, J Congleton; Western Sussex Hospitals NHS Trust, Worthing, United Kingdom

\subsection{6/thoraxjnl-2013-204457.74}

NICE $^{1}$ recommends 'track and trigger' systems for all adult acute hospital admissions and the $\mathrm{RCP}^{2}$ advocates the use of the National Early Warning Score (NEWS) to promptly highlight high risk patients. In non-selected medical patients a higher admission NEWS correlates with higher mortality, with a stepwise increase as the score increases. Anecdotally patients with COPD have high rates of NEWS alerting. No studies have looked at the validity of the NEWS in COPD patients though the issue was raised in a previous abstract.

We retrospectively interrogated an electronic observation database in our Trust (two acute sites) over a year (February 2012-January 2013). We compared acute medical unit (AMU) admissions aged over 50 years $(\mathrm{n}=13,291)$ with patients admitted with a primary diagnosis of COPD $(n=1119)$. Despite a similar age profile (median $74 \& 77$ ) and inpatient mortality 